\title{
INTEGRAL follow-up of the gravitational wave events
}

\author{
V. Savchenko*1, C. Ferrigno ${ }^{1}$, E. Kuulkers ${ }^{2}$, A. Bazzano ${ }^{3}$, E. Bozzo ${ }^{1}$, S. Brandt ${ }^{4}$, \\ J. Chenevez ${ }^{4}$, T. J.-L. Courvoisier ${ }^{1}$, R. Diehl ${ }^{5}$, A. Domingo ${ }^{6}$, L. Hanlon ${ }^{7}$, \\ E. Jourdain ${ }^{8}$, A. von Kienlin ${ }^{5}$, P. Laurent ${ }^{9,10}$, F. Lebrun ${ }^{10}$, A. Lutovinov ${ }^{11,12}$, \\ A. Martin-Carillo ${ }^{7}$, S. Mereghetti ${ }^{13}$, L. Natalucci ${ }^{3}$, J. Rodi ${ }^{3}$, J. P. Roques ${ }^{8}$, \\ R. Sunyaev ${ }^{11,14}$, and P. Ubertini ${ }^{3}$
}

${ }^{1}$ ISDC, Department of astronomy, University of Geneva, chemin d'Écogia, 16 CH-1290 Versoix, Switzerland

${ }^{2}$ European Space Research and Technology Centre (ESA/ESTEC), Keplerlaan 1, 2201 AZ Noordwijk, The Netherlands

${ }^{3}$ INAF-Institute for Space Astrophysics and Planetology, Via Fosso del Cavaliere 100, 00133-Rome, Italy

${ }^{4}$ DTU Space - National Space Institute Elektrovej - Building 327 DK-2800 Kongens Lyngby Denmark

${ }^{5}$ Max-Planck-Institut für Extraterrestrische Physik, Garching, Germany

${ }^{6}$ Centro de Astrobiología (CAB-CSIC/INTA, ESAC Campus), Camino bajo del Castillo S/N, E-28692 Villanueva de la Cañada, Madrid, Spain

${ }^{7}$ Space Science Group, School of Physics, University College Dublin, Belfield, Dublin 4, Ireland

${ }^{8}$ Université Toulouse; UPS-OMP; CNRS; IRAP; 9 Av. Roche, BP 44346, F-31028 Toulouse, France

${ }^{9}$ APC, AstroParticule et Cosmologie, Université Paris Diderot, CNRS/IN2P3, CEA/Irfu, Observatoire de Paris Sorbonne Paris Cité,

10 rue Alice Domont et Léonie Duquet, 75205 Paris Cedex 13, France.

${ }^{10}$ DSM/Irfu/Service d'Astrophysique, Bat. 709 Orme des Merisiers CEA Saclay, 91191 Gif-sur-Yvette Cedex, France

${ }^{11}$ Space Research Institute of Russian Academy of Sciences, Profsoyuznaya 84/32, 117997 Moscow, Russia

${ }^{12}$ Moscow Institute of Physics and Technology, Institutskiy per. 9, Dolgoprudny, Moscow Region, 141700, Russia

${ }^{13}$ INAF, IASF-Milano, via E.Bassini 15, I-20133 Milano, Italy

${ }^{14}$ Max Planck Institute for Astrophysics, Karl-Schwarzschild-Str. 1, Garching b. Munchen D-85741, Germany

E-mail: Volodymyr.Savchenko@unige.ch 
We use observations of the INTErnational Gamma-Ray Astrophysics Laboratory (INTEGRAL) to search for gamma-ray and hard X-ray emission associated with the gravitational wave events discovered during the first and the second scientific runs of Advanced LIGO and Advanced Virgo. The highly eccentric orbit of INTEGRAL ensures high duty cycle, long-term stable background, and unobstructed view of nearly the entire sky. This enables us to use a combination of INTEGRAL instruments (SPectrometer onboard INTEGRAL - Anti-Coincidence Shield (SPI-ACS), Imager on Board the INTEGRAL Satellite (IBIS), and IBIS/Veto) to search for a hard X-ray electromagnetic signal in the full high-probability sky region for almost every single LIGO trigger.

INTEGRAL observations of the binary black hole (BBH) mergers GW150914, LVT151012, GW170104, and GW170814 allowes to constrain the fraction of the energy promptly released in gamma-rays in $75 \mathrm{keV}-2 \mathrm{MeV}$ energy range in the direction of the observer down to as little as one millionth of the gravitational wave energy, in the majority of the localization region. Moreover, in the case of LVT151012 INTEGRAL high-energy imaging instruments, IBIS, SPectrometer onboard INTEGRAL (SPI), and Joint European X-Ray Monitor (JEM-X), provided the unique opportunity to search also for long-lasting electromagnetic counterparts of this event over 3 decades in energy, from $5 \mathrm{keV}$ to $8 \mathrm{MeV}$.

Finally, we discuss the INTEGRAL detection of the short gamma-ray burst GRB 170817A (discovered by Fermi-Gamma-ray Burst Monitor (GBM)) with a signal-to-noise ratio of 4.6, and, for the first time, its association with the gravitational waves (GWs) from binary neutron star (BNS) merging event GW170817 detected by the LIGO and Virgo observatories. The significance of association between the gamma-ray burst observed by INTEGRAL and GW170817 is $3.2 \sigma$, while the association between the Fermi-GBM and INTEGRAL detections is $4.2 \sigma$. GRB 170817A was detected by the SPI-ACS instrument about $2 \mathrm{~s}$ after the end of the gravitational wave event. We measure a fluence of $1.4 \pm 0.4 \pm 0.6 \times 10^{-7} \mathrm{erg} \mathrm{cm}^{-2}$ (75-2000 keV), where, respectively, the statistical error is given at the $1 \sigma$ confidence level, and the systematic error corresponds to the uncertainty in the spectral model and instrument response.

7th Fermi Symposium 2017

15-20 October 2017

Garmisch-Partenkirchen, Germany

\footnotetext{
*Speaker.
} 


\section{Introduction}

Gravitational waves (GWs) were predicted as a natural consequence of general relativity [1], but until recently only indirect evidence of their existence had been found by measuring the time evolution of orbital parameters in binary pulsars $[2,3]$. The first direct detection of GWs was achieved with the discovery of GW150914 [4] during the first science run of the Advanced LIGO interferometer (O1). This observation was followed by the observation of several other events of different significance [5].

The INTErnational Gamma-Ray Astrophysics Laboratory [INTEGRAL; 6] team participated in the search for electromagnetic counterparts of almost all of the LIGO/Virgo events, providing the tightest upper limits for the whole GW localization region above $75 \mathrm{keV}$. The only GW event so far during which INTEGRAL was not taking scientific data is GW151226, which happened when INTEGRAL was in the proximity of one of the perigee passages ${ }^{1}$. Slightly before and after each of these passages, all instruments on board INTEGRAL are switched off to prevent damage while crossing the Earth's radiation belts, thus limiting the observational efficiency of the observatory ("duty cycle") to $85 \%$.

\section{INTEGRAL instrument summary}

INTEGRAL [7] is an observatory with multiple instruments: a gamma-ray spectrometer $(20 \mathrm{keV}-$ $8 \mathrm{MeV}$, SPI, [8]), an imager (15 keV-10 MeV, IBIS, [9]), an X-ray monitor (3-35 keV, JEM-X, [10]), and Optical Monitoring Camera (OMC) [V band, 11].

The spectrometer SPI is surrounded by a thick Anti-Coincidence Shield (SPI-ACS). In addition to its main function of providing a veto signal for charged particles irradiating the SPI instrument, the ACS is also able to register all other impinging particles and high-energy photons. Thus, it can be used as a nearly omnidirectional detector of transient events with an effective area reaching $0.7 \mathrm{~m}^{2}$ at energies above $\sim 75 \mathrm{keV}$ and a time resolution of $50 \mathrm{~ms}$ [12]. The characterization of its response to a gamma-ray signal has been delivered with an extensive simulation study, taking into account the complex opacity pattern of materials, which are used for the INTEGRAL satellite structure and other instrument detectors. Similarly, we have computed and verified the response of the other omnidirectional detectors on board INTEGRAL: IBIS/ISGRI, IBIS/PICsIT and IBIS/Veto. For details on the INTEGRAL capabilities of detecting transients from the whole sky, particularily as relevant to our search for electro-magnetic counterparts to GW signals, we refer to [13] and references therein.

\section{Observations of the $\mathrm{BBH}$ mergers}

Electromagnetic counterparts of GW events are mainly expected if at least one neutron star is involved [see, e.g., 14, 15, 16]. However, it cannot be excluded that the merging of black holes could produce an electromagnetic signal under particular conditions ). To promote the searches for possible electromagnetic counterparts of GW events, the LIGO/Virgo collaboration distributes near real time alerts to selected teams who have signed a memorandum of understanding. These

\footnotetext{
${ }^{1}$ The INTEGRAL satellite orbital period was reduced from 3 to 2.6 sidereal days in January 2015.
} 
alerts contain a localization probability map for each event and have so far led to a massive followup campaign of GW150914, GW151226, GW170104, and GW170814 [17, 18, 19, 20, 21, 22, 23, 24, 25]. In the case of LVT151012, the localization was distributed to the community only 6 months after the event. No extensive follow-up observations have thus been performed, and only serendipitous data sets are available from all relevant facilities.

As extensively described by [13], INTEGRAL provides uniquely instantaneous coverage of the entire high-energy sky by taking advantage of the synergy between its all-sky detectors: IBIS (comprising INTEGRAL Soft Gamma-Ray Imager (ISGRI), PIxellated CsI Telescope (PICsIT), and Veto shield of the Imager on Board the INTEGRAL Satellite (IBIS/Veto)) and SPI-ACS. These provide complementary capabilities for the detection of transient events characterized by different durations, locations on the sky, and spectral energy distributions. In the case of the GW150914, GW170104, and GW170814 the most stringent upper limit on the non-detection of an electromagnetic counterpart with INTEGRAL was obtained with the SPI-ACS [26, 27], while the peculiar localization of LVT151012 [5] and its orientation with respect to the INTEGRAL satellite required the combination of the results from all detectors (together with a careful analysis of each instrument's response and background) to achieve an optimized upper limit.

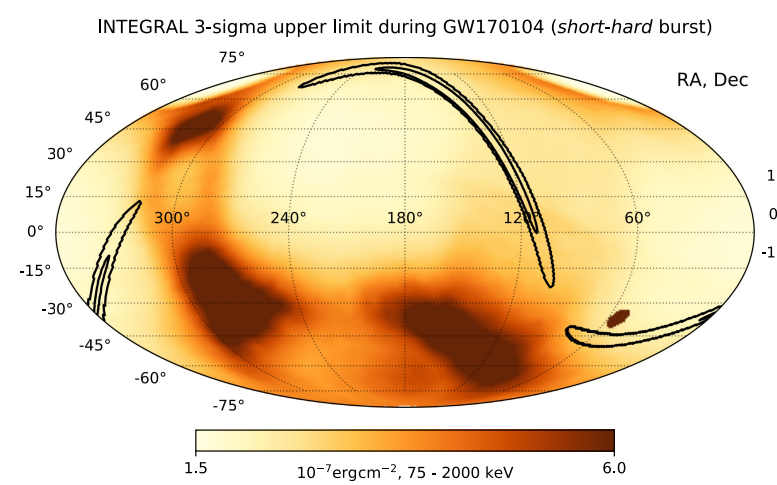

(a)

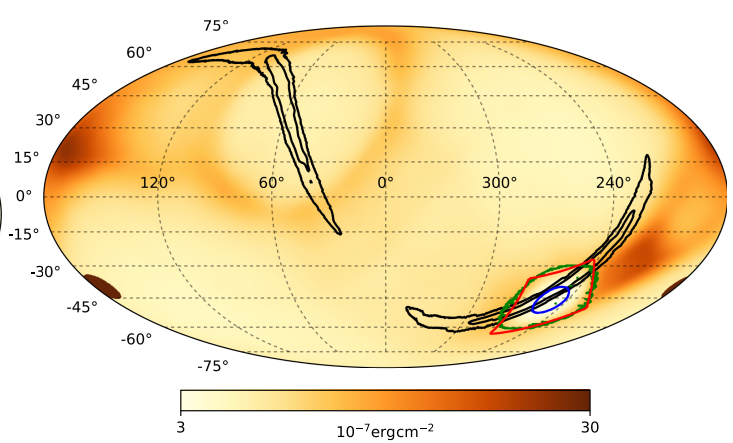

(b)

Figure 1: (a) Estimated $3 \sigma$ upper limits on the $75-2000 \mathrm{keV}$ flux of the non detected electromagnetic counterpart to GW170104 as derived from the SPI-ACS data assuming the case of a short-hard burst [see 27, for details]. The black contours show the most accurate localization of the GW event at $50 \%$ and $90 \%$ c.l., as provided by the LALInference [28].

(b) Same (a) but in the case of a long-soft [see 29, for details] burst at the time of LVT151012.

\section{GW170817 and GRB170817A}

It has long been conjectured that the subclass of gamma-ray bursts (GRBs) with a duration below about $2 \mathrm{~s}$, known as short gamma-ray bursts (sGRBs), are the product of a BNS merger and that gamma-rays are produced in the collimated ejecta following the coalescence [e.g, 30, 31, $32,33]$. So far, there was only circumstantial evidence for this hypothesis, owing to the lack of supernovae associated with sGRBs, their localization in early-type galaxies and their distinct class of duration [e.g., 34]. The advent of advanced GW detectors, which have been able to detect binary 
black hole mergers $[35,36,37,38,39]$, and have the capability to detect a signal from nearby BNS mergers [40] have sparked great expectations. Different electromagnetic signatures are expected to be associated with BNS merger events, owing to expanding ejecta, the most obvious of which is an sGRB in temporal coincidence with the GW signal and/or afterglow emission at different wavelengths in the days and/or weeks after the merger event [e.g., 41].

On n 2017 August 17 a at 12:41:04.47 UTC $\left(\mathrm{T}_{0, G W}\right.$ hereafter), a signal consistent with the merger of a BNS was detected by the LIGO-Hanford detector [42] as a single-detector trigger. The subsequent alert was issued in response to a public real-time Fermi GBM trigger on a sGRB at 12:41:06.48 UTC [43, 44, 45]; the GRB signal was immediately and independently confirmed by our team [46].

A massive follow-up campaign of the LIGO-Virgo high-probability region by optical robotic telescopes started immediately after the event and on 2017 August 18 between 1:05 and 1:45 UT, three groups reported independent detections of a transient optical source at about 10 arcsec from the center of the host S0 Galaxy NGC 4993; this source was dubbed SSS17a [47, 48] or DLT17ck [49, 50]; the transient source was confirmed by [51] [see also 52]. The source was identified as the most probable optical counterpart of the BNS merger [53, 54]. After that, it was followed at all wavelengths. The counterpart has been given the official IAU designation "AT2017gfo" [55].

The details of the detection of GRB 170817A by the INTEGRAL and the targeted followup observing campaign can be found in [56]. We also were able to search for any possible hard X-ray / soft gamma-ray emission for about six days after the prompt gamma-ray and GW signal. This allowed us to constrain both continuum emission from GRB-like afterglow emission and line emissions expected from kilonovae.

The orientation of INTEGRAL with respect to the LIGO-Virgo localization of GW170817 favored the observation by SPI-ACS and was such that the sensitivity of the IBIS was much lower in comparison [56]. For comparison of relative sensitivities of different INTEGRAL instruments see [29]. The routine untargeted search for short transients in SPI-ACS identified a single excess at $T_{0}^{\mathrm{ACS}}=T_{0}^{\mathrm{GW}}+1.88 \mathrm{~s}$ with signal-to-noise ratio $(\mathrm{S} / \mathrm{N})=4.6$ at the $0.1 \mathrm{~s}$ timescale. We derive a fluence estimate of $(1.4 \pm 0.4) \times 10^{-7} \mathrm{erg} \mathrm{cm}^{-2}$ (statistical uncertainty only) in the $75-2000 \mathrm{keV}$ energy range, consistent with GBM. The significance of the association between the GBM observation of GRB170817A and the event observed by SPI-ACS is $4.2 \sigma$. While SPI-ACS would not have alone reported this event as a gamma-ray burst (GRB), it would have reported the event while searching around GW170817, with an independent association significance of 3.2 $\sigma$ [56]. shortduration gamma-ray bursts (SGRBs) are routinely jointly detected by GBM and SPI-ACS and the association evidence from time coincidence (quoted above) as well as the consistency between the event fluences and temporal properties observed by the two instruments proves that both GBM and SPI-ACS observed the same event. The difference between the time of arrival of the signal in the SPI-ACS and GBM detectors can be exploited to improve the gamma-ray localization of GRB170817A, which may be beneficial in future joint detections.

The significant interval of the SPI-ACS lightcurve of GRB170817A is limited to a single pulse with a duration of $100 \mathrm{~ms}$ (Fig. 2a). GBM and SPI-ACS see the main pulse as appearing to have different durations because they are sensitive in different energy ranges. If the GBM data are shown in an energy range higher than the standard 50-300 keV, the main pulse is consistent with the $100 \mathrm{~ms}$ interval seen in SPI-ACS. The lightcurve observed by SPI-ACS reveals a short rise 
time $(<50 \mathrm{~ms})$ and a rapid drop $(<50 \mathrm{~ms})$. We therefore constrain the pulse duration in the energy range observed by SPI-ACS $(\sim 75-2000 \mathrm{keV})$ to less than $100 \mathrm{~ms}$.

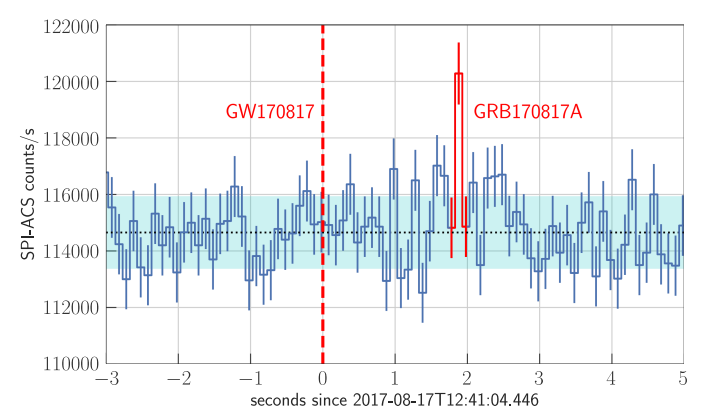

(a)

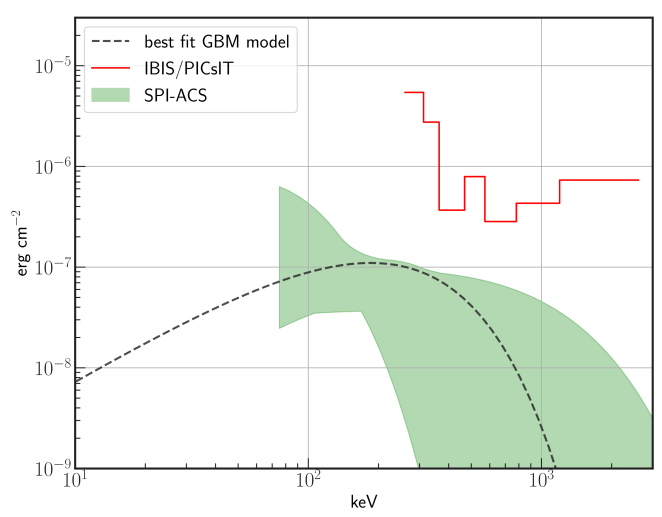

(b)

Figure 2: (a) SPI-ACS light curve of GRB 170817A (100 ms time resolution), detected 2 seconds after GW170817. The red line highlights the $100 \mathrm{~ms}$ pulse, which has an S/N of 4.6 in SPI-ACS. The blue shaded region corresponds to a range of one standard deviation of the background.

(b) Average hard X-ray/gamma-ray spectrum of the initial pulse of GRB 170817A. The shaded green region corresponds to the range of spectra compatible with the INTEGRAL/SPI-ACS observation (see text for details). IBIS/PICsIT provides a complementary independent upper limit at high energies; see text. The best-fit Fermi-GBM model for the spectrum in the same interval (Comptonized model with low-energy index of -0.62 and $E_{\text {peak }}$ of $185 \mathrm{keV}$ ) is shown as a dashed line for comparison [57].

\section{Conclusions}

Most of the GW events reported so far by LIGO were found to be most likely associated with binary back hole mergers. The extensive multi-wavelength follow-up campaigns carried out after each of these discoveries led to the detection of at least two possible electromagnetic counterparts to the GW events $[58,59]$. Although none of these associations was firmly confirmed, they led to discussion of exotic scenarios in explaining EM emission in these mergers [e.g. 60, 61, 62, 63]. The INTEGRAL efforts to follow-up as much as possible all relevant LIGO triggers will eventually help to revealing which, if any, of these scenarios is applicable. So far, the INTEGRAL results have provided the most stringent upper limits on any associated prompt hard X-ray and $\gamma$-ray emission above $\sim 75 \mathrm{keV}$ for each of the announced GW events when INTEGRAL observation was available.

Finally, we reported the independent INTEGRAL detection of a short gamma-ray burst (GRB 170817A), in coincidence with that found by Fermi-GBM [64, 65, 66] (the association significance between INTEGRAL and Fermi-GBM is 4.2 sigma), which is for the first time unambiguously associated to the gravitational wave event GW170817 observed by LIGO/Virgo and consistent with a binary neutron star merger. The joint gamma-ray and GW observation is a turning point for multi-messenger transient astrophysics. 
This observation is compatible with the expectation that a large fraction (if not all) BNS mergers might be accompanied by a prompt gamma-ray flash [66], detectable by INTEGRAL/SPI-ACS and other facilities. INTEGRAL independently detects more than 20 confirmed sGRBs per year [67]) in a broad range of fluences. With the growing sensitivity of the LIGO and Virgo observatories, being joined in the future by other observatories, we expect to detect more and more short GRBs associated with BNS mergers.

Additionally, we have exploited the unique uninterrupted serendipitous INTEGRAL observations available immediately after GRB $170817 \mathrm{~A} / \mathrm{GW} 170817$ (lasting about $20 \mathrm{ks}$ ), as well as dedicated targeted follow-up observations carried out by INTEGRAL, starting as soon as 19.5 hours after the GRB/GW (lasting in total 5.1 days). No hard X-ray or gamma-ray signal above the background was found. By taking advantage of the full sensitivity and wide FoV of the combination of the IBIS, SPI, and JEM-X instruments, we provide a stringent upper limit over a broad energy range, from $3 \mathrm{keV}$ up to $8 \mathrm{MeV}$. The INTEGRAL upper limits above $80 \mathrm{keV}$ are tighter than those set by any other instrument and constrain the isotropic-equivalent luminosity of the soft gammaray afterglow to less than $1.4 \times 10^{43} \mathrm{erg} \mathrm{s}^{-1}(80-300 \mathrm{keV})$, assuming a distance of $40 \mathrm{Mpc}$ to the source Our data exclude the possibility that a short- or a long-lasting bright hard X-ray and/or soft gamma-ray phase of activity followed GRB 170817A/GW170817.

With these results, we show that INTEGRAL continues to play a key role in the rapidly emerging multi-messenger field by constraining both the prompt and delayed gamma-ray emission associated with compact object mergers.

\section{Acknowledgements}

This work is based on observations with INTEGRAL, an ESA project with instruments and science data center funded by ESA member states (especially the PI countries: Denmark, France, Germany, Italy, Switzerland, Spain), and with the participation of Russia and the USA. The INTEGRAL SPI project has been completed under the responsibility and leadership of CNES. The SPI-ACS detector system has been provided by MPE Garching/Germany. The SPI team is grateful to ASI, CEA, CNES, DLR, ESA, INTA, NASA and OSTC for their support. The Italian INTEGRAL team acknowledges the support of ASI/INAF agreement n. 2013-025-R.1. RD and AvK acknowledge the German INTEGRAL support through DLR grant 50 OG 1101. AL and RS acknowledge the support from the Russian Science Foundation (grant 14-22-00271). AD is funded by Spanish MINECO/FEDER grant ESP2015-65712-C5-1-R. Some of the results in this paper have been derived using the HEALPix [68] package. We are grateful VirtualData from LABEX P2IO for enabling access to the StratusLab academic cloud. We acknowledge the continuous support by the INTEGRAL Users Group and the exceptionally efficient support by the teams at ESAC and ESOC for the scheduling of the targeted follow-up observations.

\section{References}

[1] A. Einstein, Näherungsweise Integration der Feldgleichungen der Gravitation, Sitzungsberichte der Königlich Preußischen Akademie der Wissenschaften (Berlin), Seite 688-696. (1916) . 
[2] R. Hulse and J. Taylor, Discovery of a pulsar in a binary system, ApJ 195 (jan, 1975) L51-L53.

[3] M. Kramer, I. Stairs, R. Manchester, M. McLaughlin, A. Lyne, R. Ferdman et al., Tests of General Relativity from Timing the Double Pulsar, Science 314 (oct, 2006) 97-102.

[4] B. P. Abbott, R. Abbott, T. D. Abbott, M. R. Abernathy, F. Acernese, K. Ackley et al., Observation of Gravitational Waves from a Binary Black Hole Merger, Physical Review Letters 116 (feb, 2016) 061102.

[5] B. P. The LIGO Scientific Collaboration, R. the Virgo Collaboration, B. P. Abbott, R. Abbott, T. D. Abbott, M. R. Abernathy et al., Binary Black Hole Mergers in the first Advanced LIGO Observing Run, Physical Review X, Volume 6, Issue 4, id.041015 6 (jun, 2016), [1606.04856].

[6] C. Winkler, T. J.-L. T. J.-L. Courvoisier, G. Di Cocco, N. Gehrels, A. Giménez, S. Grebenev et al., The INTEGRAL mission, Astronomy \& Astrophysics 411 (nov, 2003) L1-L6.

[7] C. Winkler, T. J.-L. Courvoisier, G. Di Cocco, N. Gehrels, A. Giménez, S. Grebenev et al., The INTEGRAL mission, A\&A 411 (Nov., 2003) L1-L6.

[8] G. Vedrenne, J.-P. Roques, V. Schönfelder, P. Mandrou, G. Lichti, A. von Kienlin et al., SPI: The spectrometer aboard INTEGRAL, A\&A 411 (2003) L63-L70.

[9] P. Ubertini, F. Lebrun, G. di Cocco, A. Bazzano, A. J. Bird, K. Broenstad et al., IBIS: The Imager on-board INTEGRAL, A\&A 411 (2003) L131-L139.

[10] N. Lund, C. Budtz-Jørgensen, N. J. Westergaard, S. Brandt, I. L. Rasmussen, A. Hornstrup et al., JEM-X: The X-ray monitor aboard INTEGRAL, A\&A 411 (Nov., 2003) L231-L238.

[11] J. M. Mas-Hesse, A. Giménez, J. L. Culhane, C. Jamar, B. McBreen, J. Torra et al., OMC: An Optical Monitoring Camera for INTEGRAL. Instrument description and performance, A\&A 411 (Nov., 2003) L261-L268.

[12] A. von Kienlin, V. Beckmann, A. Rau, N. Arend, K. Bennett, B. McBreen et al., INTEGRAL Spectrometer SPI's GRB detection capabilities. GRBs detected inside SPI's FoV and with the anticoincidence system ACS, A\&A 411 (Nov., 2003) L299-L305, [astro-ph/ 0308346 ].

[13] V. Savchenko, A. Bazzano, E. Bozzo, S. Brandt, J. Chenevez, T. J.-L. Courvoisier et al., INTEGRAL IBIS, SPI, and JEM-X observations of LVT151012, A\&A 603 (July, 2017) A46, [1704.01633].

[14] R. Voss and T. M. Tauris, Galactic distribution of merging neutron stars and black holes prospects for short GRB progenitors and LIGO/VIRGO, MNRAS 342 (mar, 2003) 1169-1184, [0303227].

[15] K. Siellez, M. Boer and B. Gendre, Simultaneous event detection rates by electromagnetic and gravitational wave detectors in the Advanced Era of LIGO and Virgo, MNRAS 437 (oct, 2013) 649-655, [1310.2106]. 
[16] B. Patricelli, M. Razzano, G. Cella, F. Fidecaro, E. Pian, M. Branchesi et al., Prospects for joint observations of gravitational waves and gamma rays from merging neutron star binaries, J. Cosmology Astropart. Phys. 11 (nov, 2016) 56, [1606.06124].

[17] B. Abbott, R. Abbott, T. Abbott, M. Abernathy, F. Acernese, K. Ackley et al., Localization and Broadband Follow-up of the Gravitational-wave Transient GW150914, ApJ 826 (jul, 2016) L13, [1602 . 08492$]$.

[18] B. Abbott, R. Abbott, T. Abbott, M. Abernathy, F. Acernese, K. Ackley et al., Supplement: "Localization and Broadband Follow-up of the Gravitational-wave Transient GW150914" (2016, ApJL, 826, L13), ApJS 225 (jul, 2016) 8, [1604.07864].

[19] J. Racusin, E. Burns, A. Goldstein, V. Connaughton, C. Wilson-Hodge, P. Jenke et al., Searching the Gamma-ray Sky for Counterparts to Gravitational Wave Sources: Fermi GBM and LAT Observations of LVT151012 and GW151226, ArXiv e-prints (jun, 2016), [1606.04901].

[20] M. Yoshida, Y. Utsumi, N. Tominaga, T. Morokuma, M. Tanaka, Y. Asakura et al., J-GEM follow-up observations of the gravitational wave source GW151226*, PASJ (dec, 2016), [1611.01588].

[21] A. Gando, Y. Gando, T. Hachiya, A. Hayashi, S. Hayashida, H. Ikeda et al., A Search for Electron Antineutrinos Associated with Gravitational-wave Events GW150914 and GW151226 Using KamLAND, ApJ 829 (oct, 2016) L34, [1606 . 07155].

[22] K. Abe, K. Haga, Y. Hayato, M. Ikeda, K. Iyogi, J. Kameda et al., Search for Neutrinos in Super-Kamiokande Associated with Gravitational-wave Events GW150914 and GW151226, ApJ 830 (oct, 2016) L11, [1608.08745].

[23] O. Adriani, Y. Akaike, K. Asano, Y. Asaoka, M. Bagliesi, G. Bigongiari et al., CALET Upper Limits on X-Ray and Gamma-Ray Counterparts of GW151226, ApJ 829 (sep, 2016) L20, [1607.00233].

[24] P. Cowperthwaite, E. Berger, M. Soares-Santos, J. Annis, D. Brout, D. Brown et al., A DECam Search for an Optical Counterpart to the LIGO Gravitational-wave Event GW151226, ApJ 826 (aug, 2016) L29, [1606. 04538].

[25] The Pierre Auger Collaboration, A. Aab, P. Abreu, M. Aglietta, I. Samarai, I. Albuquerque et al., Ultrahigh-energy neutrino follow-up of Gravitational Wave events GW150914 and GW151226 with the Pierre Auger Observatory, ArXiv e-prints (aug, 2016), [1608.07378].

[26] V. Savchenko, C. Ferrigno, S. Mereghetti, L. Natalucci, A. Bazzano, E. Bozzo et al., INTEGRAL upper limits on gamma-ray emission associated with the gravitational wave event GW150914, 1602.04180. 
[27] V. Savchenko, C. Ferrigno, E. Bozzo, A. Bazzano, S. Brandt, J. Chenevez et al., INTEGRAL observations of GW170104, The Astrophysical Journal Letters, Volume 846, Issue 2, article id. L23, 6 pp. (2017). 846 (jul, 2017) , [1707.03719].

[28] B. P. Abbott, R. Abbott, T. D. Abbott, F. Acernese, K. Ackley, C. Adams et al., GW170104: Observation of a 50-Solar-Mass Binary Black Hole Coalescence at Redshift 0.2, Physical Review Letters 118 (jun, 2017) 221101.

[29] V. Savchenko, A. Bazzano, E. Bozzo, S. Brandt, J. Chenevez, T. J. L. Courvoisier et al., INTEGRAL IBIS, SPI, and JEM-X observations of LVT151012, Astronomy \& Astrophysics, Volume 603, id.A46, 15 pp. $\mathbf{6 0 3}$ (apr, 2017) , [1704.01633].

[30] S. I. Blinnikov, I. D. Novikov, T. V. Perevodchikova and A. G. Polnarev, Exploding Neutron Stars in Close Binaries, Soviet Astronomy Letters 10 (Apr., 1984) 177-179.

[31] E. Nakar, Short-hard gamma-ray bursts, Phys. Rep. 442 (Apr., 2007) 166-236, [astro-ph/0701748].

[32] N. Gehrels and P. Meszaros, Gamma-Ray Bursts, Science 337 (aug, 2012) 932-936.

[33] E. Berger, Short-Duration Gamma-Ray Bursts, ARA\&A 52 (Aug., 2014) 43-105, [1311.2603].

[34] P. D'Avanzo, Short gamma-ray bursts: A review, Journal of High Energy Astrophysics 7 (2015) 73-80.

[35] Ligo Scientific Collaboration and Virgo Collaboration collaboration, B. P. Abbott, R. Abbott, T. D. Abbott, M. R. Abernathy, F. Acernese, K. Ackley et al., Observation of gravitational waves from a binary black hole merger, Phys. Rev. Lett. 116 (Feb, 2016) 061102.

[36] Ligo Scientific Collaboration and Virgo Collaboration collaboration, B. P. Abbott, R. Abbott, T. D. Abbott, M. R. Abernathy, F. Acernese, K. Ackley et al., Observation of gravitational waves from a binary black hole merger, Phys. Rev. Lett. 116 (Feb, 2016) 061102.

[37] Ligo Scientific Collaboration and Virgo Collaboration collaboration, B. P. Abbott, R. Abbott, T. D. Abbott, M. R. Abernathy, F. Acernese, K. Ackley et al., Gw151226: Observation of gravitational waves from a 22-solar-mass binary black hole coalescence, Phys. Rev. Lett. 116 (Jun, 2016) 241103.

[38] Ligo Scientific and Virgo Collaboration collaboration, B. P. Abbott, R. Abbott, T. D. Abbott, F. Acernese, K. Ackley, C. Adams et al., Gw170104: Observation of a 50-solar-mass binary black hole coalescence at redshift 0.2, Phys. Rev. Lett. 118 (Jun, 2017) 221101.

[39] LIGO/Virgo collaboration, , Phys. Rev. Lett. (2017) . 
[40] B. P. Abbott, R. Abbott, T. D. Abbott, M. R. Abernathy, F. Acernese, K. Ackley et al., Prospects for observing and localizing gravitational-wave transients with advanced ligo and advanced virgo, Living Reviews in Relativity 19 (Feb, 2016) 1.

[41] R. Fernández and B. D. Metzger, Electromagnetic Signatures of Neutron Star Mergers in the Advanced LIGO Era, Annual Review of Nuclear and Particle Science 66 (Oct., 2016) 23-45, [1512.05435].

[42] LIGO Scientific Collaboration, J. Aasi, B. P. Abbott, R. Abbott, T. Abbott, M. R. Abernathy et al., Advanced LIGO, Classical and Quantum Gravity 32 (Apr., 2015) 074001, [1411.4547].

[43] LIGO Scientific Collaboration and Virgo Collaboration, , GRB Coordinates Network 21505 (2017) .

[44] V. Connaughton, Fermi GBM trigger 170817.529 and LIGO single IFO trigger, GRB Coordinates Network 21506 (2017) .

[45] LIGO Scientific Collaboration and Virgo Collaboration, LIGO/Virgo Identification of a binary neutron star candidate coincident with Fermi GBM trigger 524666471/170817529, GRB Coordinates Network 21509 (2017) .

[46] V. Savchenko, A. Bazzano, E. Bozzo, S. Brandt, T. J.-L. Courvoisier, R. Diehl et al., , GRB Coordinates Network 21507 (2017) .

[47] D. A. Coulter, C. D. Kilpatrick, M. R. Siebert, R. J. Foley, B. J. Shappee, M. R. Drout et al., LIGO/Virgo G298048: Potential optical counterpart discovered by Swope telescope, GRB Coordinates Network 21529 (2017).

[48] D. A. Coulter and et al.in prep. (2017) .

[49] S. Yang, S. Valenti, D. Sand, L. Tartaglia, E. Cappellaro, D. Reichart et al., LIGO/Virgo G298048: DLT40 optical candidate, GRB Coordinates Network 21531 (2017) .

[50] S. Valenti, S. Yang, D. Sand, L. Tartaglia, E. Cappellaro, D. Reichart et al.in prep. (2017) .

[51] DESGW+community team, LIGO/Virgo G298048: DECam optical candidate, GRB Coordinates Network 21530 (2017).

[52] M. Soares-Santos and et alin prep. (2017).

[53] R. Foley, LIGO/Virgo G298048: Likelihood of SSS17a Being an Unrelated Extragalactic Transient in NGC 4993, GRB Coordinates Network 21557 (2017) .

[54] R. Siebert and et al.in prep. (2017) .

[55] LIGO Scientific Collaboration, Fermi-GBM, INTEGRAL and et al.ApJL (in prep.) . 
[56] V. Savchenko, C. Ferrigno, E. Kuulkers, A. Bazzano, E. Bozzo, S. Brandt et al., $<i>I N T E G R A L</ i>$ Detection of the First Prompt Gamma-Ray Signal Coincident with the Gravitational-wave Event GW170817, The Astrophysical Journal 848 (oct, 2017) L15.

[57] A. Goldstein and et al., , ApJ (2017) .

[58] V. Connaughton, E. Burns, A. Goldstein, M. Briggs, B.-B. Zhang, C. Hui et al., Fermi GBM Observations of LIGO Gravitational Wave event GW150914, ArXiv e-prints (feb, 2016), [1602.03920].

[59] F. Verrecchia, M. Tavani, A. Ursi, A. Argan, C. Pittori, I. Donnarumma et al., AGILE Observations of the Gravitational Wave Source GW170104, ArXiv e-prints (may, 2017), [1706.00029].

[60] R. Perna, D. Lazzati and B. Giacomazzo, Short Gamma-Ray Bursts from the Merger of Two Black Holes, ArXiv e-prints (feb, 2016), [1602.05140].

[61] A. Loeb, Electromagnetic Counterparts to Black Hole Mergers Detected by LIGO, ArXiv e-prints (feb, 2016), [1602.04735].

[62] S. E. Woosley, The Progenitor of GW 150914, ApJL 824 (mar, 2016) 10, [1603. 00511$].$

[63] M. Lyutikov, Fermi GBM signal contemporaneous with GW150914 - an unlikely association, eprint arXiv:1602.07352 (feb, 2016), [1602.07352].

[64] A. von Kienlin, A. Goldstein and C. Meegan, GRB 170817A: Fermi GBM detection, GCN 21520 (2017) .

[65] A. Goldstein, P. Veres, E. Burns, M. S. Briggs, R. Hamburg, D. Kocevski et al., An Ordinary Short Gamma-Ray Burst with Extraordinary Implications: $<i>$ Fermi $</ i>-G B M$ Detection of GRB 170817A, The Astrophysical Journal 848 (oct, 2017) L14.

[66] B. P. Abbott, R. Abbott, T. D. Abbott, F. Acernese, K. Ackley, C. Adams et al., Gravitational Waves and Gamma-Rays from a Binary Neutron Star Merger: GW170817 and GRB 170817A, The Astrophysical Journal Letters 848 (oct, 2017) L13.

[67] V. Savchenko, A. Neronov and T. J.-L. Courvoisier, Timing properties of gamma-ray bursts detected by SPI-ACS detector onboard INTEGRAL, A\&A 541 (May, 2012) A122, [1203.1344].

[68] K. M. Górski, E. Hivon, A. J. Banday, B. D. Wandelt, F. K. Hansen, M. Reinecke et al., HEALPix: A Framework for High-Resolution Discretization and Fast Analysis of Data Distributed on the Sphere, ApJ 622 (Apr., 2005) 759-771, [astro-ph/ 0409513 ]. 